\title{
A note on complex-4-colorability of signed planar graphs
}

\author{
Arnfried Kemnitz \\ Technical University Braunschweig \\ Braunschweig, Germany \\ a.kemnitz@tu-bs.de
}

\author{
Margit Voigt \\ University of Applied Sciences Dresden \\ Dresden, Germany \\ margit.voigt@htw-dresden.de
}

Submitted: Sep 3, 2020; Accepted: Apr 14, 2021; Published: May 7, 2021

C) The authors. Released under the CC BY-ND license (International 4.0).

\begin{abstract}
A pair $(G, \sigma)$ is called a signed graph if $\sigma: E(G) \longrightarrow\{1,-1\}$ is a mapping which assigns to each edge $e$ of $G$ a sign $\sigma(e) \in\{1,-1\}$. If $(G, \sigma)$ is a signed graph, then a complex-4-coloring of $(G, \sigma)$ is a mapping $f: V(G) \longrightarrow\{1,-1, i,-i\}$ with $i=\sqrt{-1}$ such that $f(u) f(v) \neq \sigma(e)$ for every edge $e=u v$ of $G$.

We prove that there are signed planar graphs that are not complex-4-colorable. This result completes investigations of Jin, Wong and Zhu as well as Jiang and Zhu on 4-colorings of generalized signed planar graphs disproving a conjecture of the latter authors.
\end{abstract}

Mathematics Subject Classifications: 05C10, 05C15, 05C22

\section{Introduction and results}

Let $G=(V, E)$ be a simple graph. A mapping $f: V(G) \longrightarrow[k]=\{1,2, \ldots, k\}$ ( $k$ is a positive integer) is called a $k$-coloring of $G$ if $f(u) \neq f(v)$ for every edge $e=u v$ of $G$. If such a coloring exists, then $G$ is called $k$-colorable.

A pair $(G, \sigma)$ is called a signed graph if $\sigma: E(G) \longrightarrow\{1,-1\}$ is a mapping which assigns to each edge $e$ of $G$ a sign $\sigma(e) \in\{1,-1\}$. The mapping $\sigma$ is called a signature of $G$, and an edge $e$ is positive or negative if $\sigma(e)=1$ or $\sigma(e)=-1$, respectively.

Colorings of signed graphs have been introduced in the early 1980s by Zaslavsky [4] and were later the topic of various papers (see [1] for recent informations).

Let the set $N_{k}$ be defined by $N_{k}=\left\{ \pm 1, \pm 2, \ldots, \pm \frac{k}{2}\right\}$ if $k$ is even or $N_{k}=\{0, \pm 1, \pm 2, \ldots$. $\left.\ldots, \pm \frac{k-1}{2}\right\}$ if $k$ is odd, respectively. 
Definition 1. If $(G, \sigma)$ is a signed graph and $k$ a positive integer, then a $k$-coloring of $(G, \sigma)$ is a mapping $f: V(G) \longrightarrow N_{k}$ such that $f(u) \neq \sigma(e) f(v)$ for every edge $e=u v$ of $G$. A graph $G$ is called signed $k$-colorable if $(G, \sigma)$ has a $k$-coloring for every signature $\sigma$ of $G$.

In [1] the concept of $k$-colorings of signed graphs was modified for the case $k=4$ in the following way.

Definition 2. If $(G, \sigma)$ is a signed graph, then a complex-4-coloring of $(G, \sigma)$ is a mapping $f: V(G) \longrightarrow\{1,-1, i,-i\}$ with $i=\sqrt{-1}$ such that $f(u) f(v) \neq \sigma(e)$ for every edge $e=u v$ of $G$.

It is conjectured in [1] that every signed planar graph is complex-4-colorable. We will disprove the conjecture in this note (see Theorem 6). Moreover, our result completes the investigations of Jiang and Zhu [1] on $S$ - $k$-colorability in that paper.

The concept of $S$ - $k$-colorability generalizes colorings of signed graphs and was introduced in [1] and [2]. Discussions on the relationship to other coloring concepts and a list of corresponding references can be found in these references.

The symmetric group $S_{k}$ consists of all permutations $\pi$ of $1,2, \ldots, k$. The identity permutation is written $i d$. The permutation which interchanges exactly the elements $i$ and $j$ is written by $(i j)$ and is called a cycle of length 2 . All permutations can be written as product of cycles. A set $S$ of permutations is called inverse closed if for each $\pi \in S$ also $\pi^{-1} \in S$ where $\pi^{-1}$ is the inverse permutation of $\pi$.

Definition 3. If $G=(V, E)$ is a graph and $S \subseteq S_{k}$ an inverse closed set of permutations, then an $S$-signature of $G$ is a pair $(D, \sigma)$ where $D$ is an orientation of $G$ and $\sigma: E(D) \longrightarrow$ $S$ a mapping which assigns to each $\operatorname{arc} e=(u, v)$ a permutation $\sigma(e) \in S$. A $k$-coloring of an $S$-signature of $G$ is a mapping $f: V(D) \longrightarrow[k]$ such that for each arc $e=(u, v)$ of $D$ it holds $\sigma(e)(f(u)) \neq f(v)$. A graph $G$ is called $S$ - $k$-colorable if every $S$-signature $(D, \sigma)$ of $G$ has a $k$-coloring.

Moreover, an involution is a permutation $\pi$ with $\pi^{-1}=\pi$. If all the permutations $\pi \in S$ are involutions, then the orientation of the edges is irrelevant. In our investigations we deal with involutions only. Therefore we give a further adapted definition.

Definition 4. If $G=(V, E)$ is a graph and $S \subseteq S_{k}$ a set of involutions, then an $S$ signature of $G$ is a mapping $\sigma: E(G) \longrightarrow S$ which assigns to each edge $e=u v$ an involution $\sigma(e) \in S$. A $k$-coloring of an $S$-signature of $G$ is a mapping $f: V(G) \longrightarrow[k]$ such that for each edge $e=u v$ of $G$ it holds $\sigma(e)(f(u)) \neq f(v)$. A graph $G$ is called $S$ - $k$-colorable if every $S$-signature $\sigma$ of $G$ has a $k$-coloring.

Clearly if $S=\{i d\}$, then $S$ - $k$-colorability of $G$ is equivalent to $k$-colorability of $G$. If $S=\{i d,(12)(34) \ldots((2\lfloor k / 2\rfloor-1)(2\lfloor k / 2\rfloor))\}$, then $S$ - $k$-colorability is equivalent to signed $k$-colorability. Moreover, if $S=\{(12),(34)\}$ then $S$-4-colorability is equivalent to complex-4-colorability as shown in the proof of Theorem 6 .

In [1] the question is posed for which subsets $S$ of $S_{4}$ every planar graph is $S$-4colorable. We will prove in Theorem 1 that for $S=\{(12),(34)\}$ there exist planar graphs which are not $S$-4-colorable. 
Theorem 5. If $S=\{(12),(34)\}$, then there are planar graphs that are not $S$-4-colorable.

Theorem 5 will be proved in Section 2 .

Theorem 6. There are signed planar graphs that are not complex-4-colorable.

Proof. Let $G$ be a planar graph, $S=\{(12),(34)\}$, and $\sigma$ an $S$-signature of $G$ such that $G$ is not $S$-4-colorable. Such a configuration exists according to Theorem 5 . Define a mapping $\sigma^{*}: E(G) \longrightarrow\{1,-1\}$ by

$$
\sigma^{*}(e)=\left\{\begin{array}{lll}
-1 & \text { if } & \sigma(e)=(12) \\
1 & \text { if } & \sigma(e)=(34) .
\end{array}\right.
$$

Assume to the contrary that $G$ has a complex-4-coloring $f^{*}: V(G) \longrightarrow\{1,-1, i,-i\}$ and define

$$
f(v)=\left\{\begin{array}{lll}
1 & \text { if } & f^{*}(v)=1 \\
2 & \text { if } & f^{*}(v)=-1 \\
3 & \text { if } & f^{*}(v)=i \\
4 & \text { if } & f^{*}(v)=-i
\end{array}\right.
$$

It is easy to check that $f$ is a proper $S$-4-coloring of $G$ contradicting the assumption.

In [1] a subset $S$ of $S_{4}$ is called good if every planar graph is $S$-4-colorable and the question was posed which subsets of $S_{4}$ are good. The authors mentioned that it is sufficient to restrict oneselves to subsets $S$ of $S_{4}$ where for each $a \in\{1,2,3,4\}$ there is a permutation $\pi \in S$ with $\pi(a)=a$. Such subsets are called normal subsets. If $S \in S_{4}$ is not normal then it is trivially good since there is an $a \in\{1,2,3,4\}$ with $\pi(a) \neq a$ for all $\pi \in S$ and consequently the mapping $f(v)=a$ for all $v \in V(G)$ is an $S$-4-coloring for arbitrary $G$ and arbitrary $S$-signature $\sigma$ of $G$.

It is proved in [1] that all normal subsets $S \subseteq S_{4}$ not containing $\{i d\}, S \neq S^{\prime}=$ $\{(12),(34)\}$ and $S \neq S^{\prime \prime}=\{(12),(34),(12)(34)\}$ are not good. Moreover, it is proved in [2] that if $S$ is a good subset of $S_{4}$ containing $i d$ then $S=\{i d\}$. Note that $S=\{i d\}$ is good because of the Four Color Theorem.

Summarizing these results the authors of [1] noticed that the only remaining open cases are whether $S^{\prime}$ and $S^{\prime \prime}$ are good subsets or not. Applying Theorem 5 and the results of [1] and [2] we obtain the following statement which is in some sense a strengthening of the Four Color Theorem.

Theorem 7. $S=\{i d\}$ is the only normal good subset of $S_{4}$.

\section{Proof of the main result}

At first we will proof a claim and then our main result of Theorem 5 .

Claim 8. If $S=\{(12),(34)\}$, then there is a planar graph $G$, a family of triangles $F_{1}, F_{2}, \ldots, F_{q}$ of $G$ where each $F_{i}$ is associated with a coloring $\phi_{i}$ such that the following holds: 
(1) $\phi_{i}$ colors the vertices of $F_{i}$ with three distinct colors,

(2) for any $S$-4-coloring $c$ of $G$, there is an index $i$ such that $c$ agrees with $\phi_{i}$ on $F_{i}$.

Proof. First we construct a graph $G$. Then we describe the assignment of permutations to its edges to identify the triangles $F_{i}$ and its corresponding colorings depending on the possible $S$-4-colorings $c$ of $G$.

Let $T$ be a graph consisting of two triangles $D=w_{1} w_{2} u$ and $D^{\prime}=w_{1} w_{2} u^{\prime}$ sharing the edge $w_{1} w_{2}$. If we want to color the vertices $u$ and $u^{\prime}$ with colors from $\{1,2,3,4\}$, then we have 16 possibilities: $(1,1),(1,2), \ldots,(4,4)$. For each of these cases $\left(c(u), c\left(u^{\prime}\right)\right)=(a, b)$ ( $a=b$ is possible) we take one copy of $T$, denoted by $T_{a, b}$, and construct $G$ by identifying the 16 vertices $u$ to one vertex, also denoted by $u$, and the 16 vertices $u^{\prime}$ to one vertex, also denoted by $u^{\prime}$. Therefore, $|V(G)|=34$ and $|E(G)|=80$.

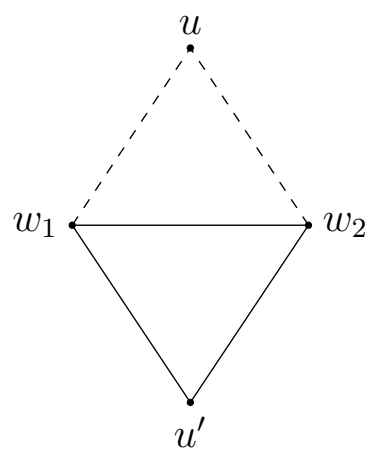

(a) $c(u)=1, c\left(u^{\prime}\right)=1$,

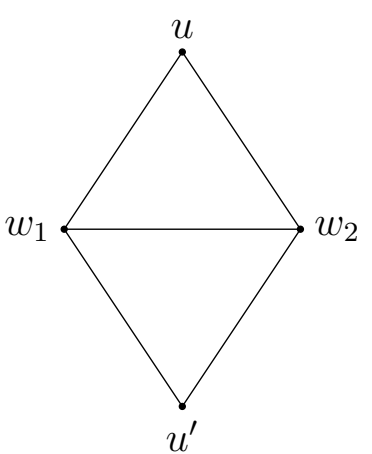

(b) $c(u)=1, c\left(u^{\prime}\right)=2$,

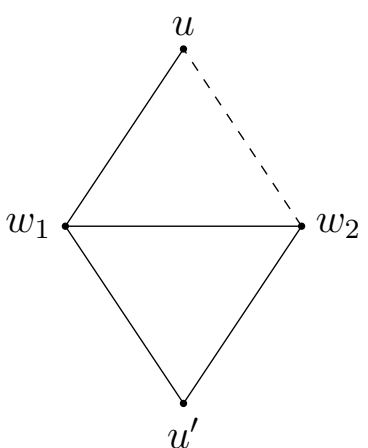

(c) $c(u)=1, c\left(u^{\prime}\right)=3$

Figure 1: Copies of $T_{a, b}$, a solid edge $e$ has assigned permutation $\sigma(e)=(12)$, a dashed edge $e$ has $\sigma(e)=(34)$.

For each of the copies $T_{a, b}$ we assign permutations to the edges depending from $a$ and $b$. We consider the possible colorings of $D$ and $D^{\prime}$ which can be created by these permutations provided that $c(u)=a$ and $c\left(u^{\prime}\right)=b$. Based on these considerations we specify colorings of the vertices of $D$ and $D^{\prime}$ given below. Note that each of these colorings uses three distinct colors and either the coloring of $D$ or the coloring of $D^{\prime}$ must occur if $c(u)=a$ and $c\left(u^{\prime}\right)=b$.

Because of symmetry it is sufficient to consider the cases $(a, b) \in\{(1,1),(1,2),(1,3)\}$ which are depicted in Figure 1.

At first assume that $c(u)=1, c\left(u^{\prime}\right)=1$ and assign $\sigma(e)$ as indicated in Figure 1(a). Then either $D=w_{1} w_{2} u$ with $c\left(w_{1}\right)=3, c\left(w_{2}\right)=4$, and $c(u)=1$ or $D^{\prime}=w_{1} w_{2} u^{\prime}$ with $c\left(w_{1}\right)=4, c\left(w_{2}\right)=3$, and $c\left(u^{\prime}\right)=1$.

Next assume that $c(u)=1, c\left(u^{\prime}\right)=2$ and permutations assigned to the edges according to Figure 1(b). Analogously, we have either $D=w_{1} w_{2} u$ with $c\left(w_{1}\right)=3, c\left(w_{2}\right)=4$, and $c(u)=1$ or $D^{\prime}=w_{1} w_{2} u^{\prime}$ with $c\left(w_{1}\right)=4, c\left(w_{2}\right)=3$, and $c\left(u^{\prime}\right)=2$. 
Finally, we assume that $c(u)=1, c\left(u^{\prime}\right)=3$ and $\sigma(e)$ as shown in Figure 1(c). Then either $D=w_{2} u w_{1}$ with $c\left(w_{2}\right)=2, c(u)=1$, and $c\left(w_{1}\right)=4$ or $D^{\prime}=u^{\prime} w_{2} w_{1}$ with $c\left(u^{\prime}\right)=3$, $c\left(w_{2}\right)=4$, and $c\left(w_{1}\right)=1$.

The family $F_{1}, F_{2}, \ldots, F_{q}$ consists of all triangles $D$ and $D^{\prime}$ where the colorings $\phi_{i}$ are given by the specified colorings indicated above. Thus Claim 8 is proved.

Proof of Theorem 5. In every triangle $F_{i}=x y z$ of $G$ we insert a structure $H_{\alpha, \beta, \gamma}$ as described below which depends on the coloring $\phi_{i}$ as indicated in the above proof. Let $G^{*}$ be the resulting graph.

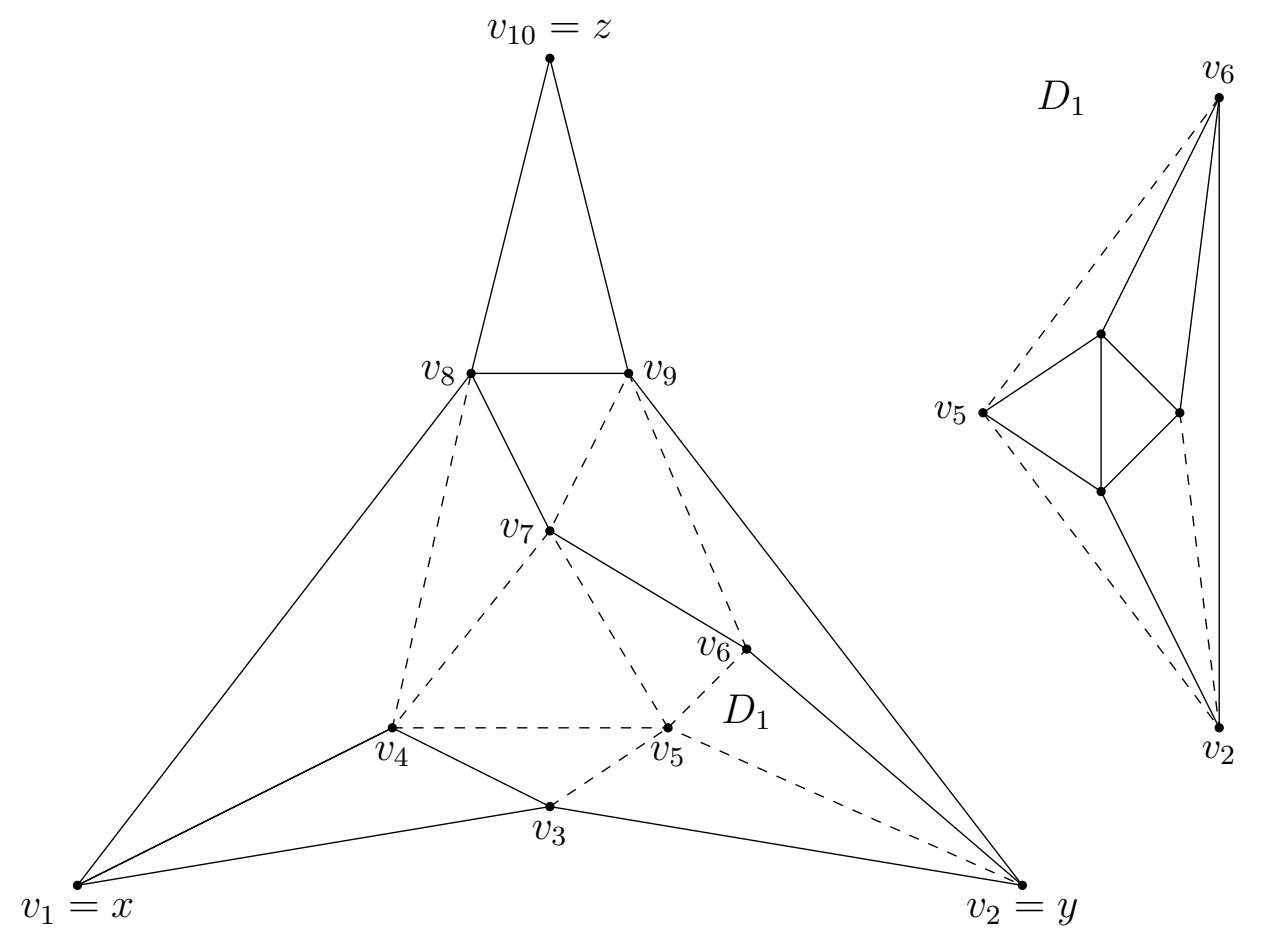

Figure 2: Subgraph $H_{1,2,3}: c\left(v_{1}\right)=1, c\left(v_{2}\right)=2, c\left(v_{10}\right)=3$, a solid edge $e$ has assigned permutation $\sigma(e)=(12)$, a dashed edge $e$ has $\sigma(e)=(34)$.

Consider the triangle $F_{i}=x y z$ with associated coloring $\phi_{i}$ where $c(x)=\alpha, c(y)=\beta$, and $c(z)=\gamma$. We will show that the precoloring of $x, y, z$ is not extendable to the inserted $H_{\alpha, \beta, \gamma}$. Note that by suitable assignment of the vertices of $F_{i}$ we may assume that $\{c(x), c(y)\}=\{1,2\}$ and $c(z) \in\{3,4\}$ or $\{c(x), c(y)\}=\{3,4\}$ and $c(z) \in\{1,2\}$. Thus, because of symmetry it is sufficient to consider

$$
c(x)=1, c(y)=2, c(z)=3 .
$$

Consider the subgraph $H_{1,2,3}$ as shown in Figure 2 with the specified permutations on the edges and assume that $v_{1}=x, v_{2}=y$, and $v_{10}=z$ with $c\left(v_{1}\right)=1, c\left(v_{2}\right)=2$, and $c\left(v_{10}\right)=3$. 
According to the assigned permutations (12) to the edges $v_{1} v_{4}$ and $v_{2} v_{5}$ color 2 is not possible for the vertices $v_{4}$ and $v_{5}$. If both $v_{4}$ and $v_{5}$ would not be colored by color 1 then $v_{4}$ and $v_{5}$ must be colored by the same color 3 or 4 because of $\sigma\left(v_{4} v_{5}\right)=(34)$. Then $v_{3}$ would not be colorable. It follows that one of the vertices $v_{4}$ and $v_{5}$ must be colored by 1 . We consider two cases.

Case 1: $c\left(v_{4}\right)=1$

Because of $c\left(v_{5}\right) \in\{3,4\}$ and $\left\{c\left(v_{6}\right), c\left(v_{7}\right)\right\} \subset\{2,3,4\}$ it follows that either $v_{6}$ or $v_{7}$ is colored by color 2 . Hence $c\left(v_{9}\right)=4$ and $v_{8}$ is not colorable.

Case 2: $c\left(v_{5}\right)=1$

Clearly, we have $c\left(v_{9}\right) \in\{2,4\}$

- $c\left(v_{9}\right)=2$ It follows that $c\left(v_{8}\right)=4$ and $c\left(v_{7}\right)=3$ which implies that $v_{4}$ is not colorable.

- $c\left(v_{9}\right)=4$ It follows that $c\left(v_{8}\right)=1, c\left(v_{7}\right)=4$, and $c\left(v_{6}\right)=2$.

Now all three vertices of the triangle inside $D_{1}$ must be colored by 3 or 4 which is not possible.

Therefore, the precoloring of $v_{1}, v_{2}$, and $v_{10}$ cannot be extended to $H_{1,2,3}$.

By Claim 8, one of the specified triangles of $G$ must be colored by the corresponding colors $\alpha, \beta, \gamma$. However, the coloring is not extendable to the inserted subgraph $H_{\alpha, \beta, \gamma}$. Thus $G^{*}$ is not S-4-colorable and the proof of Theorem 5 is complete.

Added in proof. Our main result is also contained in [3] which was presubmitted during the reviewing process of this paper.

\section{References}

[1] Y. Jiang, X. Zhu: 4-colouring of generalized signed planar graphs, The Electronic Journal of Combinatorics 27 (2020), \#P3.31.

[2] L. Jin, T. Wong, and X. Zhu: Colouring of S-labeled planar graphs, Europaen Journal of Combinatorics 92 (2021), 103198, https://doi.org/10.1016/j.ejc.2020.103198, arXiv:1811.08584v2.

[3] R. Naserasr, L. Pham: Complex and homomorphic chromatic number of signed planar simple graphs, preprint, HAL Id: hal-03000542.

[4] T. Zaslavsky: Signed graph coloring, Discrete Math. 39 (1982) 215 - 228. 